\title{
Mask-Induced Priming and the Negative Compatibility Effect
}

Petroc Sumner

Cardiff University, Cardiff, UK

\begin{abstract}
Under certain conditions, masked primes have produced counter-intuitive negative compatibility effects (NCE), such that RT is increased, not decreased, when the target is similar to the prime. This NCE has been interpreted as an index of automatic motor inhibition, triggered to suppress the partial motor activation caused by the prime. An alternative explanation is that perceptual interactions between prime and mask produce positive priming in the opposite direction to the prime, explaining the NCE without postulating inhibition. Here the potential role of this "mask-induced priming" was investigated in two experiments, using masks composed of random lines. Experiment 1 compared masks that included features of the primes and targets with masks that did not. The former should create more mask-induced priming, but the NCE did not differ between masks. Experiment 2 employed masks that contained features of either one target or the other, but not both. These asymmetric masks produced significant mask-induced priming, but it was insufficient in size to account for the prime-related NCE. Thus mask composition can contribute to NCEs, but when random line masks are employed, the major source of the NCE seems to be motor-inhibition.
\end{abstract}

Keywords: perception, action, inhibition, automatic, subliminal

Masked primes often affect responses to subsequent target stimuli, such that reaction time (RT) is reduced when the prime is similar to the target - a positive compatibility effect. However, one masked-prime paradigm has produced counter-intuitive negative compatibility effects (NCE), such that RT is increased when prime and target are similar. In a series of studies, Schlaghecken and Eimer required participants to make speeded choice responses to simple visual targets, such as leftward or rightward pointing arrows (see Eimer \& Schlaghecken, 2003, for a review). The targets were preceded by nondiscriminable masked primes that could be identical to the target (compatible) or identical to the alternative target (incompatible). When the interval between prime and target (ISI) was very short (0-60 ms), performance benefits were observed on compatible trials and performance costs on incompatible trials (a positive compatibility effect, PCE). However, when the interval was longer (100-200 ms), performance costs occurred on compatible trials and benefits on incompatible trials (a negative compatibility effect, NCE). The NCE has occurred in many studies (e.g., Aron et al., 2003; Eimer \& Schlaghecken, 2003; Klapp, 2005; Klapp \& Hinkley, 2002; Praamstra \& Seiss, 2005; Seiss \& Praamstra, 2004), but its cause has become controversial.

\section{Motor Inhibition Hypothesis}

Schlaghecken and Eimer (e.g., Eimer \& Schlaghecken, 1998; Schlaghecken, Bowman, \& Eimer, 2006; Schlaghecken \& Eimer, 2002) and Klapp and Hinkley (2002) suggested that the NCE represents an automatic self-inhibition mechanism that suppresses the partial activation initially caused by the prime. A prime associated with a leftward response initially causes some activation of the motor mechanisms leading to that response, and, through lateral inhibition, some deactivation of alternative responses. If the target is presented during this phase a PCE is predicted, as has been measured at short ISI. However, in the absence of further perceptual evidence supporting such response activation, an inhibitory phase automatically follows so that inappropriate response tendencies do not develop into actual responses. This process also releases the alternative responses from their partial deactivation through lateral inhibition. If a target is presented during this phase an NCE is predicted, as measured at longer ISI. This activation-followed-by-inhibition pattern is seen as a characteristic feature of low-level motor-control, implying that complex control processes may occur outside conscious awareness. If this account is true, it challenges the distinction between "automatic" and "controlled" processes, and also offers the masked-prime task as a method for studying automatic motor control. Indeed, various authors have already employed the paradigm with the aim of studying motor inhibition (e.g., Aron et al., 2003; Praamstra \& Seiss, 2005; Seiss \& Praamstra, 2004, 2006).

\section{Mask-Induced Priming Hypothesis}

An alternative explanation is that the NCE is produced not by inhibition of the primed response, but by positive priming of the alternative response (Lleras \& Enns, 2004; Verleger, Jas- 
kowski, Aydemir, van der Lubbe, \& Groen, 2004). In most early experiments recording an NCE, the masks were constructed by superimposing the two possible prime stimuli, leftward and rightward double arrows (e.g., Eimer \& Schlaghecken, 1998; Schlaghecken \& Eimer, 2000). Thus presenting a masked leftward prime, for example, was equivalent to presenting leftward arrows followed by rightward arrows superimposed, which could explain the reversal of the compatibility effect without the need for any inhibition. This interpretation was supported by findings that the NCE disappeared when superimposed arrow masks were replaced with masks that were not composed of features of the primes and targets (Jaskowski \& Przekoracka-Krawczyk, 2005; Lleras \& Enns, 2004; Verleger et al., 2004).

Before the studies of Verleger et al. (2004) and Lleras and Enns (2004) reached publication, several investigations (Aron et al., 2003; Eimer \& Schlaghecken, 2002; Praamstra \& Seiss, 2005; Schlaghecken \& Eimer, 2002, 2004; Seiss \& Praamstra, 2004) had measured NCEs with masks composed of random lines rather than superimposed arrows (owing to the very concerns raised by Verleger et al. and Lleras \& Enns; see footnotes 1 and 5 in Schlaghecken \& Eimer, 2002). However, while the appearance of these masks is not obviously equivalent to the appearance of the second prime, the mask-induced priming hypothesis can be extended to offer an explanation of these NCEs, because the masks still contained some features of the primes and targets (diagonal lines and arrow-like intersections). Since masks alter our perceptual representation of primes, primes may alter our perceptual representation of masks. It is common for novelty or feature differences to be exaggerated by the visual system, and therefore any features in the mask that are also in the prime may be relatively reduced in salience. This imbalance in mask feature salience could potentially cause response priming in the opposite direction from that expected from the prime, and hence create an NCE. This is my interpretation of Lleras and Enns' (2004) “object updating” and Verleger et al.'s (2004) "active mask" accounts of the NCE, which I refer to collectively as mask-induced priming (even though prime-mask interactions are involved).

\section{Relative Contribution of Mask-Induced Priming}

It is now generally accepted that masks composed of superimposed arrows (or other prime stimuli superimposed) cause significant mask-induced priming (Klapp, 2005; Lleras \& Enns, 2004, 2005; Schlaghecken \& Eimer, 2006; Verleger et al., 2004). On the other hand, Klapp (2005) and Schlaghecken and Eimer (2006) have recently found that NCEs can occur when mask-induced priming is ruled out by using masks sharing no features with the primes and targets. Thus for the many studies employing random line masks, the relative contribution of mask-induced priming remains unclear, and must be clarified if we are to conclude anything about motor inhibition from these data, and if future investigations are to employ similar paradigms. This was the aim of the current study.

Schlaghecken and Eimer (2006) found that the NCE was twice as large with random line masks as with masks composed of horizontal and vertical lines (sharing no features with the arrow primes), potentially implying that mask-induced-priming contributed about half of the NCE for the random line masks. However, the two types of mask were not equivalent in their masking effectiveness - masks sharing features with the prime are generally more effective, which is why they were originally employed. It remains debated whether there is any causal relationship between prime visibility and the NCE (Eimer \& Schlaghecken, 2002; Lleras \& Enns, 2005; Praamstra \& Seiss, 2005), but nevertheless a negative relationship between the size of the NCE and the visibility of the prime has occurred in many sets of results (Eimer \& Schlaghecken, 2002; Klapp, 2005; Klapp \& Hinkley, 2002; Lleras \& Enns, 2004, 2005; Schlaghecken \& Eimer, 2006; Sumner, Tsai, Yu, \& Nachev, 2006). Thus it is difficult to confidently assess the relative importance of mask-induced priming if the masks under comparison differ in their masking effectiveness. Therefore the experiments below were designed to compare masks that are equivalent in their ability to render the primes nondiscriminable.

\section{Methods}

\section{Design Rationale}

In Experiment 1, primes and targets were horizontal or vertical lines, and two types of masks were employed, which either did (Mask A) or did not (Mask B) contain these features (see Figure 1). Mask-induced priming predicts an NCE only for Mask A. To establish effective masking, and to keep the masks perceptually similar, each mask contained multiple line orientations chosen randomly from a set of angles. It is possible that even in Mask B a little mask-induced priming might occur from lines that were more similar to one target than the other (e.g., $22.5^{\circ}$ vs $67.5^{\circ}$ ), so to minimize this potential we used vertical and horizontal lines for the primes and targets rather than arrows, because vertical and horizontal lines are perceptually more distinct from diagonal lines than diagonal lines of equivalent angular discrepancy are from each other (Appelle, 1972). A control experiment was also performed to measure the relative priming produced by $22.5^{\circ}$ lines or $67.5^{\circ}$ lines relative to lines identical with the targets (vertical and horizontal) or neutral $45^{\circ}$ lines.

Experiment 2 was designed to deliberately introduce and measure asymmetrical response priming from mask features, to assess the size of its effect relative to the NCE. The targets were diagonal lines oriented $45^{\circ}$ to the right $\left(+45^{\circ}\right)$ 


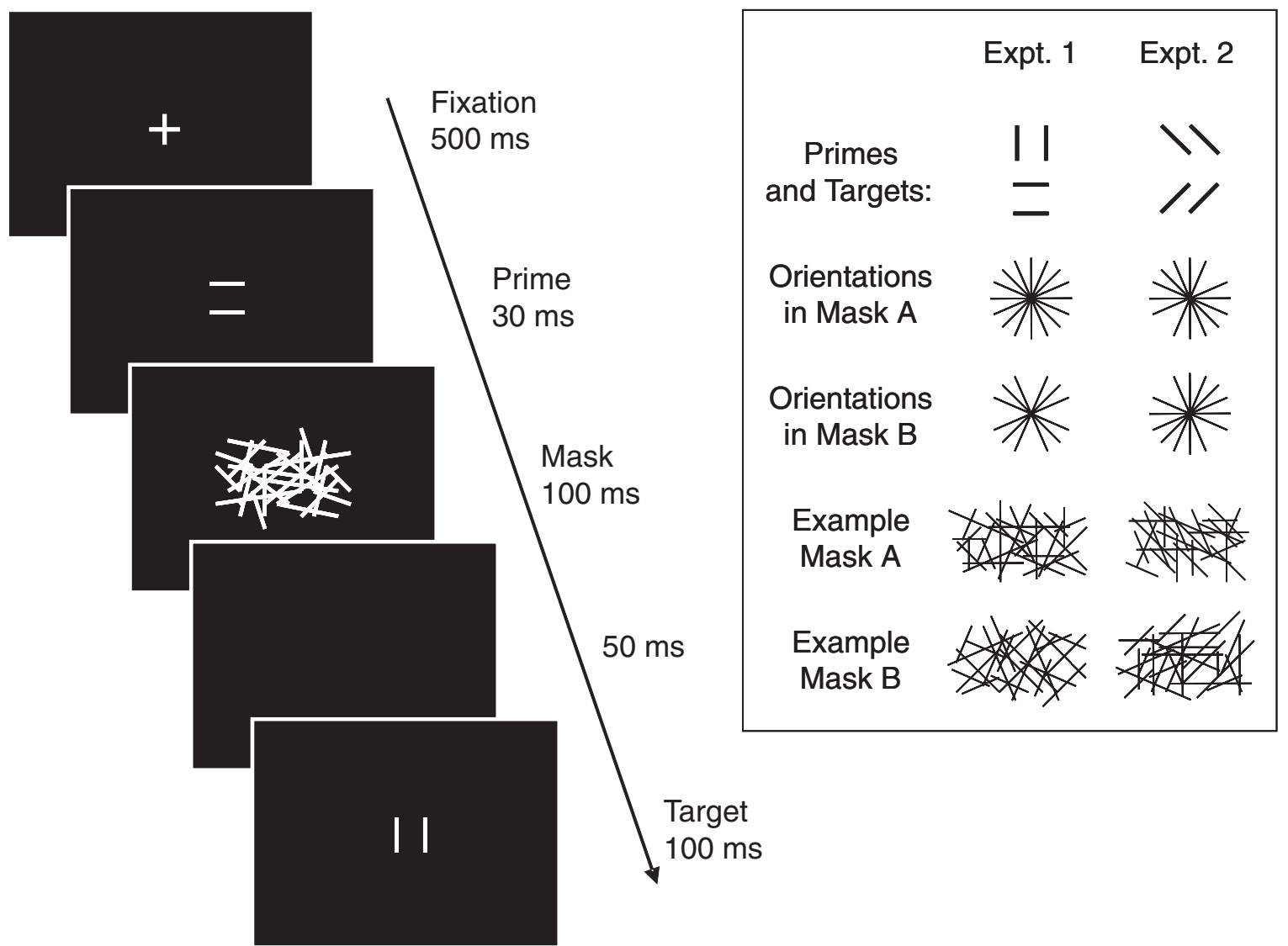

Figure 1. Schematic illustration of the procedure. One incompatible trial for Experiment 1 is shown. The right hand chart illustrates the target and prime stimuli used in each experiment, as well as example masks and the line orientations included in the masks. Note that the star-shaped schematics depict the orientations present in the masks, not the form of the masks themselves, in which the orientations were distributed randomly over a grid and each line had a random length (between limits) as illustrated in the examples.

or the left $\left(-45^{\circ}\right.$ or $\left.135^{\circ}\right)$, and two types of masks were employed, which either contained features of one target, or of the other target, but not both. Thus Mask A contained leftward $\left(135^{\circ}\right)$ lines, but not rightward $45^{\circ}$ lines, whereas Mask B contained $45^{\circ}$ but not $135^{\circ}$ lines. These asymmetric masks were expected to produce response priming, such that Mask A would speed RT to $135^{\circ}$ targets, whereas mask B would speed RT to $45^{\circ}$ targets. An NCE associated with the identity of the prime is also expected (assuming that the masks adequately mask the primes), and therefore opposite predictions are made in some types of trial for the maskinduced priming hypothesis and the motor inhibition hypothesis. For example, in trials containing a $45^{\circ}$ prime, Mask B and a $45^{\circ}$ target, inhibition of the prime would hinder performance, whereas priming from the mask would facilitate performance (see Table 1 for more examples of trials with opposite predictions).

The key question then is whether the effect of the asymmetrical masks or the effect of prime identity would be larger. In previous experiments, the masks themselves were balanced and any mask-induced priming must have resulted from interactions between primes and masks reducing the salience of features shared between prime and mask, relative to other features. It is difficult to know how much the saliency of these features was reduced, and how much priming this might cause, but we can establish an upper limit by removing the features altogether. This was the logic in Experiment 2. The physical asymmetry of target features introduced into the masks is expected to produce a positive compatibility effect between mask and target, and this effect should be more powerful than any mask-induced priming elicited by previously employed balanced random line masks. If mask-induced priming has been the main source of previous NCEs measured with random line masks, then the priming effect from these unbalanced masks should be at least as large as any NCE associated with prime identity.

\section{Participants}

14 volunteers participated in Experiment 1 (aged 22-32, 7 female). Two (one male, one female) were excluded because they perceived the primes (prime discrimination per- 
formance of $94 \%, d^{\prime}=2.20$, and $\left.83 \%, d^{\prime}=1.35\right)$, whereas we had aimed to make the primes nondiscriminable (maximum discrimination performance among other participants was $66 \%, d^{\prime}=0.58$, with a mean of $54 \%, d^{\prime}=0.14$ ). 8 volunteers participated in the control experiment related to Experiment 1 (aged 19-33, 6 female). 12 volunteers (aged 21-34, 4 female) participated in Experiment 2. All participants had normal or corrected-to-normal acuity.

\section{Apparatus}

Stimulus presentation employed a Cambridge Research Systems (CRS) ViSaGe and a 21-inch Sony GDM-F520 Trinitron monitor. Stimulus presentation was synchronized with the screen refresh rate of $100 \mathrm{~Hz}$, and timings were controlled and measured by the CRS clock (and not subject to the errors produced by normal PC operating systems). Manual responses were made with a CRS CB6 button box. Viewing distance was maintained at $70 \mathrm{~cm}$ by a chin rest.

\section{Stimuli and Procedure}

Each trial consisted of a prime, a mask and a target. Targets were pairs of parallel lines $1^{\circ}$ long and $1^{\circ}$ apart (Figure 1), which could be either vertical or horizontal (Experiment 1), or tilted $45^{\circ}$ to the left or right (Experiment 2). Participants responded as quickly as possible by pressing a left or right button (stimulus-response mappings were counterbalanced across subjects). The prime was either identical to the target (compatible) or the stimulus associated with the opposite response (incompatible). The mask was approximately $2^{\circ}$ by $3^{\circ}$ and composed of 30 lines of random length (between limits) and centered on a $6 \times 5$ grid covering the prime stimulus. In Experiment 1, Mask A contained orientations of $0^{\circ}$ (vertical), $22.5^{\circ}, 45^{\circ}, 67.5^{\circ}, 90^{\circ}$ (horizontal), $112.5^{\circ}, 135^{\circ}$ or $157.5^{\circ}$. Mask B contained only six of these eight angles: $22.5^{\circ}, 45^{\circ}, 67.5^{\circ}, 112.5^{\circ}, 135^{\circ}$ or $157.5^{\circ}$, so that no vertical or horizontal lines (the target features) were included. In Experiment 2, Mask A contained orientations of $0^{\circ}, 22.5^{\circ}, 67.5^{\circ}$, $90^{\circ}, 112.5^{\circ}, 135^{\circ}$ and $157.5^{\circ}$, but no $45^{\circ}$ lines. Mask B contained $0^{\circ}, 22.5^{\circ}, 45^{\circ} 67.5^{\circ}, 90^{\circ}, 112.5^{\circ}$, and $157.5^{\circ}$, but no $135^{\circ}$ lines.

The prime was displayed for two fames $(20 \mathrm{~ms})$, and after a blank frame $(10 \mathrm{~ms})$ the mask appeared for 10 frames $(100 \mathrm{~ms})$. The target followed mask offset after 5 frames $(50 \mathrm{~ms})$ and was displayed for $100 \mathrm{~ms}$. Following the response there was $1000 \mathrm{~ms}$ pause, a fixation cross for $500 \mathrm{~ms}$ and a blank of $300 \mathrm{~ms}$ at the start of the new trial.

There was no predictable relationship between the prime, mask and target: each participant performed 400 trials comprising 50 of each of the 8 trial types (left/right targets; compatible/incompatible primes; MaskA/B) in a random order, preceded by 80 practice trials. Participants were instructed to respond as quickly and accurately as possible to the targets and ignore the previous stimuli. Reaction times (RT) below $200 \mathrm{~ms}$ or above $800 \mathrm{~ms}$ were considered anticipations or lapses, respectively, and automatically deleted. Error responses were removed from the RT analysis. For each participant, mean and median RT was obtained for the 8 trial types, which were collapsed into compatible and incompatible conditions for each type of mask.

Following the main experiment, perceptual awareness (discrimination performance) of the primes was measured in trials where the targets were omitted. Each participant was asked to report prime identity (forced choice, nonspeeded) in 80 trials (stimulus-response mappings for each participant were the same as in the main experiment).

In the control experiment related to Experiment 1, participants responded to horizontal and vertical targets identical to those in Experiment 1. There were eight possible prime stimuli corresponding to the eight line orientations used in the masks for Experiment 1 (and there were no masks). Each prime contained two lines of that orientation (length $1^{\circ}$ ), and was displayed for 5 frames. The target followed after 10 blank frames (producing the same interval as the mask-target interval in Experiment 1).

\section{Results}

\section{Experiment 1 - Control Experiment}

Mean RTs for targets with identically oriented primes, neutral primes $\left(45^{\circ}\right)$ and incompatible primes were $407 \mathrm{~ms}$, $497 \mathrm{~ms}$ and $543 \mathrm{~ms}$, respectively, showing a very large compatibility effect. Mean RTs for the crucial trials with primes oriented $22.5^{\circ}$ or $67.5^{\circ}$ away from the target orientation were $480 \mathrm{~ms}$ and $508 \mathrm{~ms}$, respectively, lying as expected between the neutral condition and the compatible or incompatible conditions, respectively. Importantly, the primes oriented $22.5^{\circ}$ away from the target produced significantly different results from the fully compatible primes $\left(t_{(7)}=10.5, p<.001\right)$, and the difference of $73 \mathrm{~ms}$ between these two types of prime was significantly greater than the difference of $17 \mathrm{~ms}$ between the $22.5^{\circ}$ primes and the neutral $\left(45^{\circ}\right)$ primes $\left(t_{(7)}=4.1, p<.01\right)$. Likewise the primes oriented $67.5^{\circ}$ away from the target orientation produced significantly different results from the fully incompatible primes $\left(t_{(7)}=3.4, p=.01\right)$, and the difference of $35 \mathrm{~ms}$ between these two types of prime was significantly greater than the difference of $11 \mathrm{~ms}$ between the $67.5^{\circ}$ primes and the neutral $\left(45^{\circ}\right)$ primes $\left(t_{(7)}=2.9, p<.05\right)$. Therefore, in the case of vertical and horizontal targets, $22.5^{\circ}$ lines and $67.5^{\circ}$ lines appear to be more similar in their priming properties to $45^{\circ}$ lines than to vertical and horizontal lines. This being the case, the mask-induced priming hypothesis should predict a large difference in the NCE for masks A and B in Experiment 1, since only Mask A contained horizontal and vertical lines. 


\section{Experiment 1 - Main Experiment \\ Discrimination of the Prime}

Mean discrimination performance was $54 \%\left(d^{\prime}=0.14\right)$ for Mask A (containing vertical and horizontal lines) and 53\% $\left(d^{\prime}=0.11\right)$ for mask B (not containing vertical and horizontal lines), neither of which differs significantly from chance (Mask A, $t_{(11)}=0.8, p=.43$; Mask B, $t_{(11)}=1.46, p=.17$; combined, $\left.t_{(11)}=1.41, p=.19\right)$. Some participants reported noticing that some masks contained target features, which, if anything, we might expect to boost mask-induced priming.

\section{Compatibility Effects}

For mean RT there was a main effect of compatibility $(F(1,11)=38, M S E=69, p<.001)$, but no interaction between this and mask-type $(F(1,11)<1)$. The experiment had a power of $0.8(\beta=0.2)$ to detect a significant difference between the NCEs for each mask of $6.9 \mathrm{~ms}$. Crucially, the NCEs for both masks were clearly reliable on their own (Mask A, $t_{(11)}=4.4, p<.01$; Mask B, $t_{(11)}=7.0, p<.001$ ), and were only $1.1 \mathrm{~ms}$ different from each other (Figure 2). The standard error of this difference was $2.8 \mathrm{~ms}$ and the 95\% confidence bounds were $5.0 \mathrm{~ms}$ (Mask A > Mask B) and $7.2 \mathrm{~ms}$ (Mask B > Mask A). In other words, although Mask B, which contained no target features, should produce little if any mask-induced priming, the true mean NCE for Mask B is unlikely to be more than $5 \mathrm{~ms}$ smaller than the mean NCE for Mask A. A $t$ test confirmed that the difference between the NCEs for each mask was much smaller than the mean size of the NCE itself $\left(t_{(11)}=5.8, p\right.$ $<.001$; this pattern was found for all participants but one).

For median RT, the same pattern was apparent: a main effect of compatibility $(F(1,11)=31, M S E=83, p<.001)$, no interaction with mask-type $(F(1,11)<1)$, and robust NCEs for both masks alone (Mask A, $t_{(11)}=5.5, p<.001$; Mask B, $\left.t_{(11)}=4.5, p<.001\right)$. The difference between the NCEs for each mask was only $1.3 \mathrm{~ms}$, with standard error of $2.5 \mathrm{~ms}$ and a $95 \%$ confidence bound of $6.9 \mathrm{~ms}$ in the direction of the mask-induced priming hypothesis (Mask A > Mask B). Equivalent patterns in error rate were not significant.

There was no correlation across participants between prime discrimination performance and the size of the NCE for Mask A $(r=-0.28 ; p=.4)$ or Mask B $(r=-0.08 ; p=.8$; but when all participants have near chance prime discrimination, there is not much scope for a correlation to occur).

\section{Experiment 2}

\section{Discrimination of the Prime}

Mean discrimination performance was $49 \%$ for Mask A and $52 \%$ for mask B, which do not statistically differ from chance (both $\left.t_{(11)}<1\right)$. One participant had a score of $67.5 \%\left(d^{\prime}=\right.$ $\left.0.64, \chi^{2} p<.05\right)$, while all others scored below $58 \%\left(d^{\prime}=\right.$

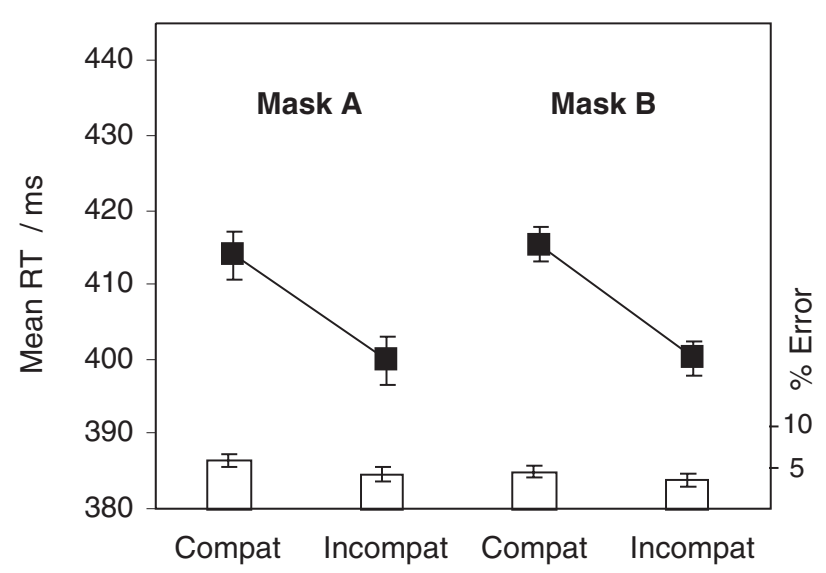

Figure 2. Results for Experiment 1. Filled symbols and lines represent mean RT for compatible and incompatible trials (averaged across left and right targets), while open bars represent error rates. The mask-induced priming hypothesis predicts a negative compatibility effect (NCE) for Mask A, but not for Mask B, which did not contain any target features (vertical and horizontal lines). The motor inhibition hypothesis predicts NCEs for both types of mask. Error bars show standard errors of the within subject compatibility effects.

0.29). No participant reported noticing the difference between the masks, but when the mask shared elements with the prime (Mask A with $135^{\circ}$ primes and Mask B with $45^{\circ}$ primes), mean correct was $56 \%$, whereas when the mask contained elements of the alternative prime, mean performance was $45 \%\left(t_{(11)}=3.8, p<.01\right)$. Better prime discrimination for masks containing prime features might possibly account for some of the apparent mask compatibility effect described below (examine Table 1), making mask-induced priming even less important. However, given that masks containing prime features are expected to be more, not less, effective masks (e.g., Schlaghecken \& Eimer, 2006), and that discrimination with masks containing the alternative prime features was significantly below $50 \%\left(t_{(11)}=2.2, p<.05\right)$, it is likely that the features in the mask simply biased discrimination responses, rather than truly altered prime visibility.

\section{Compatibility Effects}

Mean RT in trials with compatible primes was greater than mean RT in trials with incompatible primes $\left(t_{(11)}=7.4, p<\right.$ .001 ): a prime-target NCE of $25.8 \mathrm{~ms}$ (Table 1; Figure 3). NCEs also occurred in median RT $\left(26.5 \mathrm{~ms} ; t_{(11)}=7.1, p<\right.$ $.001)$ and in the error rates $\left(3.1 \% ; t_{(11)}=4.0, p<.01\right)$. The effect of the mask was also evident: mean RT was $11.6 \mathrm{~ms}$ faster for masks containing target features than for masks containing features of the opposite target $\left(t_{(11)}=5.4, p<\right.$ $.001)$, and this pattern was also mirrored in median RT $\left(12.8 \mathrm{~ms} ; t_{(11)}=5.3, p<.001\right)$ and in the error rates $(1.6 \%$; $\left.t_{(11)}=4.3, p<.01\right)$. ANOVAs with factors of prime com- 


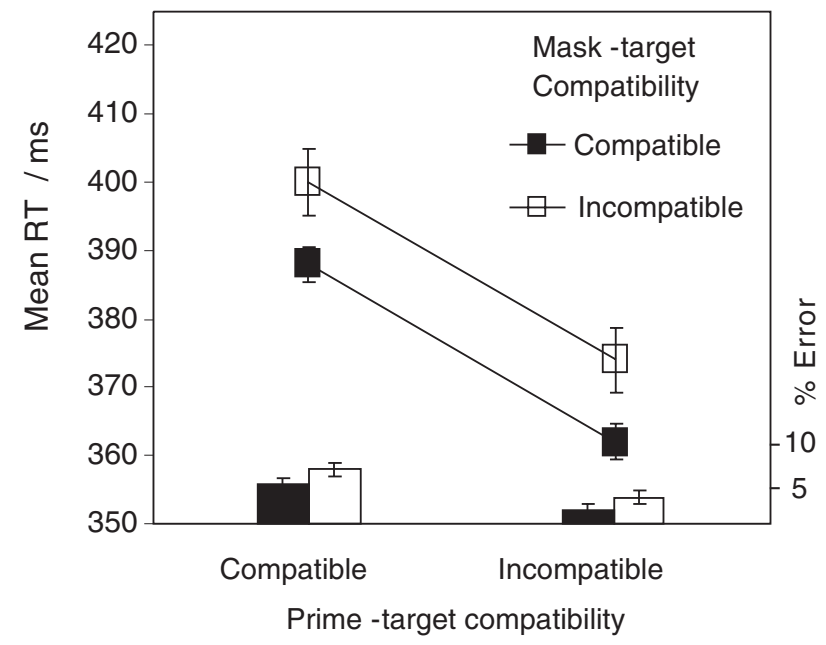

Figure 3. Prime-Target compatibility effects and Mask-Target compatibility effects for Experiment 2. As in Figure 2, the lines plot RT, while the bars plot error rates. Prime-target compatibility refers to whether the prime was the same as the target, or associated with the opposite response. Mask-target compatibility refers to whether the mask contained features of that trial's target, or of the alternative target. The key findings are that both a prime-target NCE and a mask-target compatibility effect occurred, but the latter was much smaller than the former, making it implausible that mask induced priming can account for the whole NCE. Error bars are standard errors of the NCEs for each condition of mask compatibility. patibility and mask compatibility confirmed the main effects for both these factors in mean RT $(F(1,11)=23, M S E$ $=259, p=.001 ; F(1,11)=19, M S E=71, p=.001)$, median RT $(F(1,11)=21, M S E=303, p=.001 ; F(1,11)=23$, $M S E=79, p=.001)$, and in error rates $(F(1,11)=12, M S E$ $=8.3, p<.01 ; F(1,11)=10, M S E=2.2, p<.01)$. There were no interactions (all $F$ values $<1.7$ ). There was no correlation across participants between prime discrimination performance and the size of the NCE $(r=-0.07 ; p=$ .8 ) or the mask compatibility effect $(r=0.03 ; p=.9$; but when all participants have near chance prime discrimination, there is not much scope for a correlation to occur).

Crucially, the main effect of mask compatibility was only half as large as the NCE associated with the primes (Figure 3). Moreover, the NCE was larger than the mask compatibility effect for the whole $95 \%$ confidence interval of this difference (5.6 to $22.8 \mathrm{~ms}$ for means; 4.2 to $23.3 \mathrm{~ms}$ for medians; $t$-test comparisons of the numerical size of the effects: mean RT, $t_{(11)}=3.6, p<.01$; median RT, $t_{(11)}=3.2$, $p<.01$; error rate, $\left.t_{(11)}=1.7, p>.05\right)$. Thus, while there was a measurable priming effect from the mask, it was not nearly large enough to account for the whole NCE.

Note that the design of Experiment 2 allowed the data to be labeled according to three different compatibility variables: prime-target compatibility, mask-target compatibility and prime-mask compatibility. However, only two of the three are independent (the third variable is the interaction of the other two), so that whichever two are chosen, the data reduce to the same four values (see Figure 3): $388 \mathrm{~ms}$ for compatible prime-target, mask-target and

Table 1. Trial types, predictions and results for Experiment 2

\begin{tabular}{|c|c|c|c|c|c|c|c|c|}
\hline Trial type & 1 & 2 & 3 & 4 & 5 & 6 & 7 & 8 \\
\hline Target & 11 & // & 11 & $/ /$ & 11 & $/ /$ & 11 & $/ /$ \\
\hline Prime & 11 & // & // & 11 & 11 & $/ /$ & // & 11 \\
\hline Prime-Target compatibility & compat & compat & incompat & incompat & compat & compat & incompat & incompat \\
\hline Mask & contains $\backslash$ & contains $\backslash$ & contains $\backslash$ & contains $\backslash$ & contains // & contains // & contains // & contains // \\
\hline Mask-Target compatibility & compat & incompat & compat & incompat & incompat & compat & incompat & compat \\
\hline \multicolumn{9}{|l|}{ Predictions: } \\
\hline Motor inhibition & inhibition & inhibition & facilitation & facilitation & inhibition & inhibition & facilitation & facilitation \\
\hline Mask-induced priming & facilitation & inhibition & facilitation & inhibition & inhibition & facilitation & inhibition & facilitation \\
\hline \multicolumn{9}{|l|}{ Results: } \\
\hline Mean RT (ms) & 388 & 395 & 363 & 377 & 400 & 386 & 374 & 364 \\
\hline Median RT (ms) & 383 & 386 & 353 & 371 & 394 & 378 & 367 & 353 \\
\hline Error Rate (\%) & 6.3 & 6.2 & 1.5 & 3.8 & 5.7 & 2.8 & 2.3 & 1.8 \\
\hline
\end{tabular}

The trial type section shows the orientation of the targets and primes for each of the eight trial types, and whether the mask contained lines of that orientation or not. The motor inhibition hypothesis predicts that motor activation by the prime is inhibited, so responses to a compatible target will be inhibited and responses to the opposite target will be facilitated. Mask-induced priming predicts that when the mask contains elements of the target, responses are facilitated, and when the mask contains elements of the opposite target, responses are inhibited. If the prime interacts with the mask to make prime features less salient, then mask-induced priming also predicts a similar pattern as the motor inhibition hypothesis, but this pattern should be weaker than the one produced directly by the features actually present in the mask. Both patterns are present in the results (see Figure 3). The results shown are the means of each participant's mean RT, median RT and error rate per condition. 
prime-mask; $362 \mathrm{~ms}$ for incompatible prime-target, compatible mask-target and incompatible prime-mask; $400 \mathrm{~ms}$ for compatible prime-target, incompatible mask-target and incompatible prime-mask; $374 \mathrm{~ms}$ for incompatible primetarget, incompatible mask-target and compatible primemask. If instead of using prime-target and mask-target variables for the analysis, as above, we use prime-target and prime-mask factors for the ANOVA, there is no main effect of prime-mask compatibility, but there is an interaction. This occurs because the prime-mask variable represents the interaction between prime-target compatibility and masktarget compatibility, while the interaction between primemask and prime-target is the main effect of mask-target compatibility (reported above). Therefore, while the data can be described in different ways, the results are the same: a large effect of prime-target compatibility (the NCE), a smaller effect of mask-target compatibility, and no interaction between them (i.e., no main effect of prime-mask compatibility). However, the choice of variables used to describe the data can imply different interpretations, and this issue is discussed below.

\section{Discussion}

\section{Mask-Induced Priming}

If masked prime paradigms are to be used to study mechanisms of motor initiation and control, it is important to understand the potential influences of any interactions between the stimuli employed. If the mask alters our perceptual representation of the prime, then the prime may alter our perceptual representation of the mask, and any resultant saliency imbalance for features similar to the targets could potentially cause patterns of response activation opposite to, or different from, those predicted from the primes (Jaskowski \& Przekoracka-Krawczyk, 2005; Lleras \& Enns, 2004; Verleger et al., 2004). I have referred to this general possibility as mask-induced priming, to distinguish it from the traditional idea of prime-induced priming.

It is now generally accepted that mask-induced priming is important when masks are constructed by combining the features of each prime (Klapp, 2005; Lleras \& Enns, 2004; Schlaghecken \& Eimer, 2002; Verleger et al., 2004). Here, I have attempted to clarify the role of mask-induced priming when masks are composed of randomly oriented lines, as used in many studies that have purported to study motor inhibition (e.g., Aron et al., 2003; Eimer \& Schlaghecken, 2002; Praamstra \& Seiss, 2005; Schlaghecken \& Eimer, 2002, 2004; Seiss \& Praamstra, 2004, 2006).

Mask-induced priming predicts an NCE for masks containing the features of the primes and targets, but not for masks that don't contain these features. However, Experiment 1 found NCEs for both types of mask, and any difference between these NCEs was likely to be less than $5 \mathrm{~ms}$ (according to the $95 \%$ confidence interval of the difference between the measured NCEs). In Experiment 2, mask-induced priming was produced by a physical imbalance of features in the masks, which was expected to represent an upper limit of the likely perceptual imbalance that might occur through saliency modulation of any mask features that are similar to prime features. However, the effect of the physically imbalanced masks was only half as large as the NCE associated with prime identity. Taken together, the results of the two experiments suggest that when random line masks are employed, mask-induced priming is not the only factor producing the NCE. Indeed, in the conditions employed here, it appears to make only a minor contribution.

It is important to consider whether the main effects of prime-target compatibility and mask-target compatibility measured in Experiment 2 were direct causal effects of the prime and mask, respectively, on responses to the target, or whether they might have arisen through indirect effects of prime-mask compatibility. In other words, the mask-target compatibility effect might have arisen through the mask modulating the prime-target compatibility effect, rather than the mask directly affecting responses to the target (and in this case the mask-target main effect would be better described as the interaction between prime-mask compatibility and primetarget compatibility). In fact, this possibility has already been raised in the context of prime visibility (see "Discrimination of the Prime"): if prime-mask compatibility modulated prime visibility, this could in turn modulate the prime-target NCE. However, if the mask-target compatibility effect can be accounted for in this or other ways, this acts only to reduce the impact of mask-induced priming even further, in line with the conclusions already reached above that it is not the major source of the NCEs measured here.

More pertinent to the current discussion is whether the prime-target compatibility effect should more appropriately be considered an interaction between prime-mask compatibility and mask-target compatibility. In fact, this is in essence an expression of the mask-induced priming hypothesis, and therefore this issue has also already been discussed above in different words. If we assume no direct influence of the prime on responses to the target, we must explain how prime identity can modulate the mask-target compatibility effect by plus or minus $26 \mathrm{~ms}$, while the mask-target compatibility effect itself was only $12 \mathrm{~ms}$. Is it plausible that saliency modulation or contrast effects produced by a $20 \mathrm{~ms}$ prime on a $100 \mathrm{~ms}$ mask could be so much larger than the effect of actually including or omitting target features in the mask itself? It would seem much more plausible to accept that, in addition to any such mask-induced priming effects, the prime also affects responses to the targets via motor activation and inhibition.

\section{Source Confusion and Feature Discounting}

The results reported here also argue against an alternative explanation of the NCE involving an extension of Huber et al.'s (Huber, Shiffrin, Lyle, \& Ruys, 2001; Huber, Shiffrin, 
Quach, \& Lyle, 2002) computational theory for "responding optimally with unknown sources of evidence" (ROUSE). Huber et al. argued that when targets and primes are present in temporal proximity, there is source confusion between the features activated by each, and participants employ a cognitive mechanism of feature "discounting" to attempt to remove prime activation from the target decision process. If this discounting process over-compensates for prime activity, then a bias against targets that share these features can occur (i.e., an NCE). Although, Huber et al. found over-compensation only for long duration visible primes, in the context of the masked-prime paradigm, features of the visible mask may be subjected to the discounting process, and assuming source confusion between activation from prime and mask, prime features may also get discounted (J. Enns, personal communication).

However, if the NCE is to be explained by over-discounting of prime activity source-confused with the mask, this would predict that features of the mask itself should also be over-discounted, leading to a negative, not positive, masktarget compatibility effect in Experiment 2. Indeed, Huber et al. (2001, 2002) explicitly predicted that over-discounting was more likely for more strongly activated features. Thus the co-occurrence of a prime-target NCE and a mask-target positive compatibility effect in the same experiment appears to rule out an explanation of the NCE along these lines.

\section{Prime-Target Perceptual Interactions}

Lleras and Enns (2005) suggested that, in addition to primemask interactions, prime-target interactions may contribute to the NCE by rendering targets opposite to the prime more salient (though processes akin to repetition blindness or negative priming). They found an NCE when target and prime occurred in the same location, but a PCE when they occurred in different locations. However, attention to the primed location presumably differed across these conditions, and attention has been found to modulate the effect of primes in this paradigm (Sumner et al., 2006) and in others (Lachter, Forster, \& Ruthruff, 2004; Naccache, Blandin, \& Dehaene, 2002). Other studies (which used random line masks) have found robust NCEs with primes and targets presented in different locations (Praamstra \& Seiss, 2005; Seiss \& Praamstra, 2004; Sumner et al., 2006). Moreover, in a control experiment, Sumner et al. found equivalent NCEs for targets presented in the same or different location as the prime (19 ms vs $20 \mathrm{~ms}$ ). Thus the current evidence suggests that while perceptual prime-target interactions may occur, they do not account for the NCE measured in most studies.

\section{Motor Inhibition}

If perceptual interactions between primes and masks or primes and targets cannot account for the whole NCE when random line masks are employed, then motor inhibition seems to make an important contribution. However, the mechanism by which inhibition occurs may not follow the hypothesis developed by Schlaghecken and Eimer (e.g., 1998, 2002, 2006) and by Klapp and Hinkley (2002), in which partially activated responses self-inhibit when perceptual evidence supporting them is removed (see Sumner, 2007 , for a review of recent evidence for and against components of this motor inhibition theory). It has recently been proposed that motor inhibition may instead be stimulus-triggered. In the "mask-triggered inhibition" hypothesis (Jaskowski, 2007; Jaskowski \& Przekoracka-Krawczyk, 2005) and the "onset-triggered suppression" hypothesis (Lleras \& Enns, 2006) the appearance of a second stimulus after the prime - the mask - automatically elicits an "emergency break" or "whoops response" that inhibits any motor activation initiated by the prime. In support of this idea, NCEs were found with "masks" that did not spatially overlap the primes, and thus did not remove perceptual evidence of the primes (Jaskowski, 2007; Lleras \& Enns, 2006). Thus, even in circumstances where motor inhibition makes the main contribution to the NCE, masks may still play an essential role beyond simply reducing prime visibility.

\section{Pragmatics of Mask Composition}

It is clear that to investigate automatic motor inhibition with masked primes we must design masks with care. It is important to control both mask-induced priming and prime visibility, because the latter's potential relationship with the NCE is yet to be clarified. It is very difficult to obtain effective masking when masks contain no features more similar to one target than to the other, but one way forward might be to use masks like Mask B in Experiment 1. This type of mask contains several orientations (or other features), but omits the features most similar to the primes and targets that are most likely to cause mask-induced priming. Any residual mask-induced priming should be small given that there was no evidence of it in Experiment 1 even with Mask A, which included target-like features.

An important aspect of the random line masks employed in this and other studies is that new random masks were generated on each trial (e.g., Schlaghecken \& Eimer, 2002, 2004, 2006). The original reasoning for this was to avoid increased prime discrimination due to perceptual learning of the mask, but it may also be important in reducing consistent mask-induced priming. If the motor priming under investigation requires consistent mapping between simple stimuli and specific responses, it makes sense that denying such consistency from the masks may reduce their potential priming ability.

\section{Acknowledgments}

I thank Tracey Brandwood for help with the control experiment, Liz Coulthard and Parashkev Nachev for comments, 
Jim Enns for the extension of Huber et al.'s ROUSE theory (2001, 2002), and all the participants for their time. The work was supported by the BBSRC and the School of Psychology, Cardiff University.

\section{References}

Appelle, S. (1972). Perception and discrimination as a function of stimulus orientation: The "oblique effect" in man and animals. Psychological Bulletin, 78, 266-278.

Aron, A.R., Schlaghecken, F., Fletcher, P.C., Bullmore, E.T., Eimer, M., Barker, R. et al. (2003). Inhibition of subliminally primed responses is mediated by the caudate and thalamus: Evidence from functional MRI and Huntington's disease. Brain, 126, 713-723.

Eimer, M., \& Schlaghecken, F. (1998). Effects of masked stimuli on motor activation: Behavioral and electrophysiological evidence. Journal of Experimental Psychology: Human Perception and Performance, 24, 1737-1747.

Eimer, M., \& Schlaghecken, F. (2002). Links between conscious awareness and response inhibition: Evidence from masked priming. Psychonomic Bulletin and Review, 9, 514-520.

Eimer, M., \& Schlaghecken, F. (2003). Response facilitation and inhibition in subliminal priming. Biological Psychology, 64, 7-26.

Huber, D.E., Shiffrin, R.M., Lyle, K.B., \& Ruys, K.I. (2001). Perception and preference in short-term word priming. Psychological Review, 108, 652.

Huber, D.E., Shiffrin, R.M., Quach, R., \& Lyle, K.B. (2002). Mechanisms of source confusion and discounting in short-term priming: 1. Effects of prime duration and prime recognition. Memory and Cognition, 30, 745-757.

Jaskowski, P. (2007). The effect of nonmasking distractors on the priming of motor responses. Journal of Experimental Psychology: Human Perception and Performance, 33, 456-468.

Jaskowski, P., \& Przekoracka-Krawczyk, A. (2005). On the role of mask structure in subliminal priming. Acta Neurobiologiae Experimentalis, 65, 409-417.

Klapp, S.T. (2005). Two versions of the negative compatibility effect: Comment on Lleras and Enns (2004). Journal of Experimental Psychology: General, 134, 431-435.

Klapp, S.T., \& Hinkley, L.B. (2002). The negative compatibility effect: Unconscious inhibition influences reaction time and response selection. Journal of Experimental Psychology: General, 131, 255-269.

Lachter, J., Forster, K.I., \& Ruthruff, E. (2004). Forty-five years after Broadbent (1958): Still no identification without attention. Psychological Review, 111, 880-913.

Lleras, A., \& Enns, J.T. (2004). Negative compatibility or object updating? A cautionary tale of mask-dependent priming. Journal of Experimental Psychology: General, 133, 475-493.

Lleras, A., \& Enns, J.T. (2005). Updating a cautionary tale of masked priming: Reply to Klapp (2005). Journal of Experimental Psychology: General, 134, 436-440.

Lleras, A., \& Enns, J.T. (2006). How much like a target can a mask be? Geometric, spatial, and temporal similarity in priming: A reply to Schlaghecken and Eimer (2006). Journal of Experimental Psychology: General, 135, 495-500.
Naccache, L., Blandin, E., \& Dehaene, S. (2002). Unconscious masked priming depends on temporal attention. Psychological Science, 13, 416-424.

Praamstra, P., \& Seiss, E. (2005). The neurophysiology of response competition: Motor cortex activation and inhibition following subliminal response priming. Journal of Cognitive Neuroscience, 17, 483-493.

Schlaghecken, F., Bowman, H., \& Eimer, M. (2006). Dissociating local and global levels of perceptuo-motor control in masked priming. Journal of Experimental Psychology: Human Perception and Performance, 32, 618-632.

Schlaghecken, F., \& Eimer, M. (2000). A central-peripheral asymmetry in masked priming. Perception and Psychophysics, 62, 1367-1382.

Schlaghecken, F., \& Eimer, M. (2002). Motor activation with and without inhibition: Evidence for a threshold mechanism in motor control. Perception and Psychophysics, 64, 148-162.

Schlaghecken, F., \& Eimer, M. (2004). Masked prime stimuli can bias "free" choices between response alternatives. Psychonomic Bulletin and Review, 11, 463-468.

Schlaghecken, F., \& Eimer, M. (2006). Active masks and active inhibition: A comment on Lleras and Enns (2004) and on Verleger, Jaskowski, Aydemir, van der Lubbe, and Groen (2004). Journal of Experimental Psychology: General, 135, 484-494.

Seiss, E., \& Praamstra, P. (2004). The basal ganglia and inhibitory mechanisms in response selection: Evidence from subliminal priming of motor responses in Parkinson's disease. Brain, 127, 330-339.

Seiss, E., \& Praamstra, P. (2006). Time-course of masked response priming and inhibition in Parkinson's disease. Neuropsychologia, 44, 869-875.

Sumner, P. (2007). Negative and positive masked-priming - implications for motor inhibition. Advances in Cognitive Psychology, 3, 317-326.

Sumner, P., Tsai, P.-C., Yu, K., \& Nachev, P. (2006). Attentional modulation of sensorimotor processes in the absence of perceptual awareness. Proceedings of the National Academy of Science USA, 103, 10520-10525.

Verleger, R., Jaskowski, P., Aydemir, A., van der Lubbe, R.H., \& Groen, M. (2004). Qualitative differences between conscious and nonconscious processing? On inverse priming induced by masked arrows. Journal of Experimental Psychology: General, 133, 494-515.

Received September 21, 2006

Revision received March 12, 2007

Accepted March 12, 2007

\section{Petroc Sumner}

School of Psychology

Cardiff University

Tower Building

Park Place

Cardiff CF10 3AT

UK

E-mail sumnerp@cardiff.ac.uk 